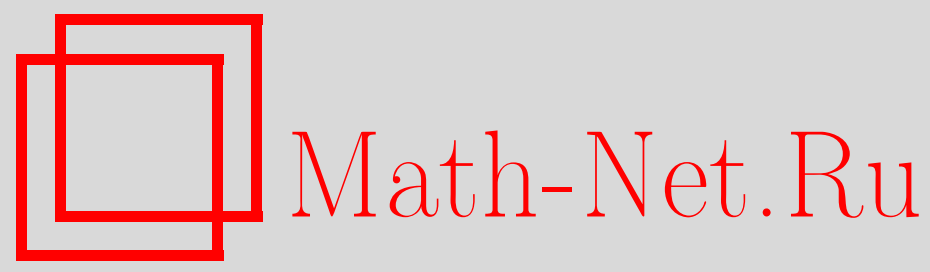

В. З. Гринес, В. С. Медведев, Е. В. Жужома, О поверхностных аттракторах и репеллерах на 3-многообразиях, Maтем. заметки, 2005, том 78, выпуск 6, 813-826

DOI: https://doi.org/10.4213/mzm2655

Использование Общероссийского математического портала Math-Net.Ru подразумевает, что вы прочитали и согласны с пользовательским соглашением http://www.mathnet.ru/rus/agreement

Параметры загрузки:

IP : 54.237 .59 .107

26 апреля 2023 г., 12:52:11

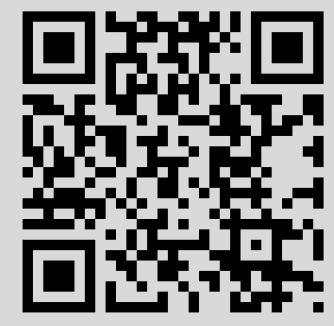




\section{О ПОВЕРХНОСТНЫХ АТТРАКТОРАХ И РЕПЕЛЛЕРАХ}

\section{НА 3-МНОГООБРАЗИЯХ}

\section{В. З. Гринес, В. С. Медведев, Е. В. Жужома}

Мы показьваем, что если $f: M^{3} \rightarrow M^{3}$ является $A$-диффеоморфизмом с поверхностным двумерным аттрактором или репеллером $\mathscr{B}$, для которого $M_{\mathscr{B}}^{2}$ является носителем, тогда $\mathscr{B}=M_{\mathscr{B}}^{2}$ и существует $k \geqslant 1$ такое, что

1) $M_{\mathscr{B}}^{2}$ есть объединение $M_{1}^{2} \cup \cdots \cup M_{k}^{2}$ непересекающихся ручных поверхностей таких, что каждая поверхность $M_{i}^{2}$ гомеоморфна двумерному тору $T^{2}$;

2) ограничение $f^{k}$ на $M_{i}^{2}, i \in\{1, \ldots, k\}$, сопряжено с автоморфизмом Аносова на торе $T^{2}$.

Библиографоия: 28 названий.

1. Введение. Одним из важных вопросов теории динамических систем является изучение взаимосвязи между рассматриваемьм классом систем и топологией несущих многообразий. Например, Франкс [1] и Ньюхаус [2] показали, что любой аносовский диффеоморфизм коразмерности 1 сопряжен с гиперболическим автоморфизмом тора (как следствие, многообразие, допускающее такие диффеоморфизмы, гомеоморфно тору $\left.T^{n}\right)$. Простое доказательство этого результата было недавно получено в работе [3] с помощью техники теории слоений.

Гринес и Жужома показали в [4] (результат анонсирован в [5]), что если замкнутое $n$-многообразие $M^{n}, n>2$, допускает структурно устойчивьй диффеоморфизм $f$ с ориентируемьм растягивающимся аттрактором коразмерности 1 , то многообразие $M^{n}$ гомотопно эквивалентно тору $T^{n}$ и гомеоморфно $T^{n}$ для $n \neq 4$. Кроме того, неблуждающее множество диффеоморфизма $f$ не содержит нетривиальных базисных множеств, отличных от растягивающегося аттрактора коразмерности один. Это позволило классифицировать (с точностью до сопряженности) структурно устойчивые диффеоморфизмы с растягивающимися аттракторами и сжимающимися репеллерами коразмерности один на $T^{n}$.

Отправной точкой для получения упомянутых выше результатов является существование так называемой гиперболической структуры на неблуждающем множестве диффеоморфизма. Более точно, пусть $f: M \rightarrow M$ является диффеоморфизмом замкнутого $k$-многообразия $M, k=\operatorname{dim} M \geqslant 2$, наделенного некоторой римановой метрикой $\rho$ (все

Работа выполнена при частичной поддержке Российского фонда фундаментальных исследований, гранты № 02-01-00098, № 05-01-00501. 
определения этого пункта могут быть найдены в [6] и [7]). Точка $x \in M$ является неблуждающей, если для любой окрестности $U$ точки $x$ пересечение $f^{n}(U) \cap U \neq \varnothing$ для бесконечного множества целых $n$. Тогда неблуждающее множество $N W(f)$ определяется как множество неблуждающих точек и является $f$-инвариантным и замкнутьп. Замкнутое инвариантное множество $\Lambda \subset M$ называется әиперболическим, если существует непрерьвное $d f$-инвариантное разложение касательного подрасслоения $T_{\Lambda} M$ в сумму $E_{\Lambda}^{s} \oplus E_{\Lambda}^{u}$ устойчивого и неустойчивого подрасслоений таких, что вьполняются оценки

$$
\left\|d f^{n}(v)\right\| \leqslant C \lambda^{n}\|v\| \quad \forall v \in E_{\Lambda}^{s}, \quad\left\|d f^{-n}(w)\right\| \leqslant C \lambda^{n}\|w\| \quad \forall w \in E_{\Lambda}^{u}, \quad \forall n \in \mathbb{N},
$$

для некоторых фиксированных чисел $C>0$ и $\lambda<1$.

Для каждого $x \in \Lambda$ множества

$$
\begin{aligned}
& W^{s}(x)=\left\{y \in M: \lim _{j \rightarrow \infty} \rho\left(f^{j}(x), f^{j}(y)\right) \rightarrow 0\right\}, \\
& W^{u}(x)=\left\{y \in M: \lim _{j \rightarrow \infty} \rho\left(f^{-j}(x), f^{-j}(y)\right) \rightarrow 0\right\}
\end{aligned}
$$

являются гладкими инъективно вложенными подмногообразиями; при этом $E_{x}^{s}$ и $E_{x}^{u}$ являются касательными пространствами к $W_{x}^{s}$ и $W_{x}^{u}$ соответственно. Множества $W^{s}(x)$ и $W^{u}(x)$ назьваются соответственно устойчивым и неустойчивым многообразием точки $x$.

Для диффеоморфизма $f: M \rightarrow M$ Смейл [8] ввел аксиому $A$ : множество $N W(f)$ является гиперболическим и периодические точки плотны в $N W(f)$. Диффеоморфизмы, удовлетворяющие аксиоме $A$, назьваются $A$-диффеоморфизмами. Согласно теореме Смейла о спектральном разложении множество $N W(f)$ любого $A$-диффеоморфизма $f$ представляется в виде конечного объединения непересекающихся так называемых базисных множеств $\Omega_{1}, \ldots, \Omega_{k}$ таких, что каждое $\Omega_{i}$ является замкнутым, $f$-инвариантным и содержит плотную орбиту [8]. Следуя [9], пару $(a, b)$ назьвают типом базисного множества $\Omega$, если $a=\operatorname{dim} E_{x}^{s}$ и $b=\operatorname{dim} E_{x}^{u}$, где $x \in \Omega$.

Смейл привел несколько важных примеров базисных множеств различной топологической размерности:

(a) нулевой размерности - подковы Смейла;

(b) размерности один - соленоиды Смейла и их обобщение - соленоиды СмейлаВильямса (см. [10], название предложено в [11]);

(с) коразмерности один - растягивающиеся аттракторы и сжимающиеся репеллеры DA-диффеоморфизмов;

(d) базисные множества транзитивных аносовских диффеоморфизмов, чьи размерности совпадают с размерностями несущего многообразия (потому что они совпадают с этим многообразием).

Хорошо известно, что нет ограничений на топологию несущего многообразия, когда базисное множество $\mathscr{B}$ имеет нулевую размерность [12]. В случае ненулевой размерности базисного множества, ситуация, вообще говоря, другая, о чем говорят упомянутые выше примеры. В дополнение к ним отметим работу [13], в которой доказано, что неблуждающее множество $A$-диффеоморфизма замкнутого ориентируемого 
3 -многообразия $M$ содержит заузленньй соленоид Смейла тогда и только тогда, когда разложение $M$ в связную сумму имеет линзовое пространство $L(p, q)$ с $p \neq 0, \pm 1$ в качестве простого слагаемого ${ }^{1}$. Недавно этот результат был передоказан в [14].

Настоящая статья посвящена топологической классификации поверхностных базисных множеств $A$-диффеоморфизмов на гладких замкнутых ориентируемых 3 -многообразиях.

Введем понятие поверхностного базисного множества.

ОПреДЕЛЕниЕ 1 . Базисное множество $\mathscr{B} A$-диффеоморфизма $f: M^{3} \rightarrow M^{3}$ назьвается поверхностным базисным множеством, если $\mathscr{B}$ принадлежит $f$-инвариантной замкнутой поверхности $M_{\mathscr{B}}^{2}$, топологически вложенной в 3 -многообразие $M^{3}$.

$f$-инвариантная поверхность $M_{\mathscr{B}}^{2}$ назьвается носителем для $\mathscr{B}$.

По определению носитель не обязан быть связным. Однако существует некоторая степень диффеоморфизма $f$, для которой каждое поверхностное базисное множество имеет связный носитель.

Напомним, что базисное множество $\mathscr{B} A$-диффеоморфизма $f: M \rightarrow M$ назьвается аттрактором, если существует замкнутая окрестность $U$ множества $\mathscr{B}$ такая, что

$$
f(U) \subset \operatorname{int} U, \quad \bigcap_{j \geqslant 0} f^{j}(U)=\mathscr{B} .
$$

Если $\mathscr{B}$ является двумерньм базисньм множеством $A$-диффеоморфизма $f$, заданного на замкнутом 3 -многообразии $M^{3}$, то согласно [16, теорема 3] $\mathscr{B}$ является либо аттрактором, либо репеллером.

Напомним, что аттрактор $\mathscr{B} A$-диффеоморфизма $f$, заданного на замкнутом многообразии $M$, назьвается растягивающимся аттрактором, если топологическая размерность $\operatorname{dim} \mathscr{B}$ равна размерности $\operatorname{dim}\left(E_{\mathscr{B}}^{u}\right)$ неустойчивого подрасслоения $E_{\mathscr{B}}^{u}$ (название предложено в [10], [17]). Сжимающийся репеллер диффеоморфизма $f$ является растягиваюшимся аттрактором для $\left.f^{-1}\right)$. Ясно, что изучение сжимающегося репеллера аналогично изучению растягивающегося аттрактора и наоборот. Хорошо известно, что растягивающийся аттрактор коразмерности 1 состоит из $(\operatorname{dim} M-1)$-мерных неустойчивых многообразий $W^{u}(x), x \in \mathscr{B}$, и локально гомеоморфен прямому произведению

${ }^{1}$ Пусть $M, M_{1}, M_{2}$ - связные 3-многообразия. Напомним, что $M$ является связной суммой $M_{1}$ и $M_{2}$ (обозначается $M=M_{1} \# M_{2}$ ), если существуют замкнутые 3 -диски $B_{i} \subset M_{i}$ и вложения $h_{i}: M_{i}-\operatorname{Int} B_{i} \rightarrow M, i=1,2$, такие, что

$$
\begin{gathered}
h_{1}\left(M_{1}-\operatorname{Int} B_{1}\right) \cap h_{2}\left(M_{2}-\operatorname{Int} B_{2}\right)=h_{1}\left(\partial B_{1}\right)=h_{2}\left(\partial B_{2}\right), \\
M=h_{1}\left(M_{1}-\operatorname{Int} B_{1}\right) \cup h_{2}\left(M_{2}-\operatorname{Int} B_{2}\right) .
\end{gathered}
$$

При этом $M_{1}, M_{2}$ называются слагаемыми. 3-многообразие $M$ называется простым, если из условия $M=M_{1} \# M_{2}$ следует, что в точности одно многообразие из $M_{1}$ и $M_{2}$ должно быть гомеоморфно сфере. Хорошо известно (см., например, [15, теорема 3.15]), что каждое компактное 3-многообразие может быть представлено как связная сумма конечного числа простых слагаемых. Напомним, что линзовое пространство является простым многообразием (см. [13, упражнение 3.12]). 
$(\operatorname{dim} M-1)$-мерного евклидового пространства и канторовского множества (см., например, [16, теорема 2]). Аналогичную структуру имеет сжимающийся репеллер коразмерности 1. Поэтому употребляется унифицирующеепонятие псевдоручного базисного множества для растягиваюшегося аттрактора или сжимающегося репеллера.

Насколько мы знаем, является открытым следующий вопрос Смейла (см. [8, с. 785]): существует ли базисное множество коразмерности один, которое не является компактным подмногообразием и не устроено локально как прямое произведение евклидового пространства и канторовского множества?

В п. 2 мы докажем (в лемме 1), что поверхностньй аттрактор (соответственно репеллер) $\mathscr{B}$ размерности два $A$-диффеоморфизма $f: M^{3} \rightarrow M^{3}$ имеет тип $(2,1)$ (соответственно $(1,2))$. Отсюда следует, что $\mathscr{B}$ не является ни растягивающимся аттрактором, ни сжимающимся репеллером.

Кроме того, мы докажем в п. 2 (лемма 2), что двумерное базисное множество $\mathscr{B}$ совпадает с его носителем $M_{\mathscr{B}}^{2}$.

Таким образом, вопрос Смейла в рассматриваемом нами случае может быть сформулирован следующим образом: существует ли двумерньй аттрактор (соответственно репеллер) с типом $(2,1)$ (соответственно $(1,2))$, отличный от компактного подмногообразия?

Заметим, что согласно [18] существует пример $A$-диффеоморфизма, заданного на замкнутом 3-многообразии, неблуждающее множество которого состоит из двумерного поверхностного базисного множества, носитель которого, вообще говоря, является не гладко вложенным 2-тором. Отметим также, что согласно [19], [20] существует диффеоморфизм Морса-Смейла $f: S^{3} \rightarrow S^{3}$, обладающий $f$-инвариантной притягивающей поверхностью $S$, которая гомеоморфна сфере $S^{2}$ и дико вложена в $S^{3}$ (следует подчеркнуть, что $S$ не является в этом случае базисным множеством диффеоморфизма $f$ ).

Однако первый результат нашей статьи утверждает, что носитель поверхностного базисного множества является объединением ручньх торов.

Напомним определение ручной поверхности, вложенной в $M^{3}$.

Пусть $D_{0}=\left\{(x, y, z) \in \mathbb{R}^{3} \mid x^{2}+y^{2} \leqslant 1, z=0\right\}$ - стандартньй диск, и $M^{2}$ - поверхность, топологически вложенная в 3 -многообразие $M^{3}$. Поверхность $M^{2}$ назьвается локально плоской или ручной, если для каждой точки $x \in M^{2}$ существует окрестность $U_{x}$ точки $x$ в $M^{3}$ и гомеоморфизм $h_{x}: \overline{U_{x}} \rightarrow \mathbb{R}^{3}$ такой, что $h\left(\overline{U_{x} \cap M^{2}}\right)=D_{0}$.

Теорема 1. Пусть $f: M^{3} \rightarrow M^{3}$ является $A$-диффеоморфизмом с поверхностным двумерным базисным множеством $\mathscr{B}$ и $M_{\mathscr{B}}^{2}$ - носитель для $\mathscr{B}$. Тогда $\mathscr{B}=M_{\mathscr{B}}^{2}$ и существует число $k \geqslant 1$ такое, что $M_{\mathscr{B}}^{2}$ является обтединением $M_{1}^{2} \cup \cdots \cup M_{k}^{2}$ непересекаюш,ихся ручных поверхностей, гомеоморфных тору $T^{2}$.

Следующая теорема описьвает динамику ограничения диффеоморфизма $f$ на поверхностное базисное множество.

ТЕОрема 2. Пусть выполнены условия теоремы 1. Тогда существует число $k \geqslant 1$ такое, что ограничение $f^{k}$ на $M_{i}^{2}, i \in\{1, \ldots, k\}$, сопряжено с аносовским автоморфизмом тора $T^{2}$.

2. Доказательство теоремы 1. Доказательство теоремы 1 следует из лемм 1,2 и 3 , которые будут доказаны ниже. 
ЛЕмма 1. Пусть Я̈ является двумерным поверхностным аттрактором (соответственно репеллером) А-диффеоморфизма $f$, заданного на 3-многообразии $M^{3}$. Тогда $\mathscr{B}$ имеет тип $(2,1)$ (соответственно $(1,2)$ ).

ДокАЗАТЕЛЬСТво. Предположим для определенности, что $\mathscr{B}$ - поверхностный аттрактор и $M_{\mathscr{B}}^{2}-$ носитель для $\mathscr{B}$. Согласно [16, теорема 1] для любой точки $z \in \mathscr{B}$ неустойчивое многообразие $W^{u}(x)$ принадлежит $\mathscr{B}$. Так как ограничение $f$ на $\mathscr{B}$ транзитивно, то $\operatorname{dim} W^{u}(z)$ не зависит от выбора $z \in \mathscr{B}$. Напомним, что $\operatorname{dim} E_{z}^{u}=\operatorname{dim} W^{u}(z)$. Таким образом, достаточно доказать что $\operatorname{dim} W^{u}(z)=1$.

Предположим противное. Так как $\operatorname{dim} W^{u}(z) \leqslant \operatorname{dim} \mathscr{B}$, то либо $\operatorname{dim} W^{u}(z)=0$, либо $\operatorname{dim} W^{u}(z)=2$.

Если $\operatorname{dim} W^{u}(z)=0$, тогда $\mathscr{B}$ должно быть притягивающей орбитой диффеоморфизма $f$ и, следовательно, нульмерным базисньм множеством. Это противоречит предположению о том, что $\operatorname{dim} \mathscr{B}=2$.

Предположим, что $\operatorname{dim} W^{u}(z)=2$. Так как $\mathscr{B}$ - нетривиальное базисное множество, то оно содержит бесконечное множество $\operatorname{Per}(f)_{\mathscr{B}}$ периодических точек, которое плотно в $\mathscr{B}$. С другой стороны, так как $\mathscr{B}$ является поверхностньм базисным множеством, то для любой точки $p \in \operatorname{Per}(f)_{\mathscr{B}}$ неустойчивое многообразие $W^{u}(p)$ принадлежит поверхности $M_{\mathscr{B}}^{2}$ и, следовательно, множество $W^{u}(p) \backslash\{p\}$ не содержит периодических точек диффеоморфизма $f$. Это противоречит тому, что множество $\operatorname{Per}(f)_{\mathscr{B}}$ плотно в $\mathscr{B}$.

Пусть $\mathscr{B}$ - двумерньй аттрактор $A$-диффеоморфизма $f: M^{3} \rightarrow M^{3}$.

Согласно Аносову [21] и Боуэну [22] существует число $k \geqslant 1$ такое, что базисное множество $\mathscr{B}$ может быть представлено как объединение замкнутых непересекающихся подмножеств $\mathscr{B}_{1}, \ldots, \mathscr{B}_{k}$ таких, что

$$
f\left(\mathscr{B}_{i}\right)=\mathscr{B}_{i+1} \quad\left(\mathscr{B}_{k+1}=\mathscr{B}_{1}\right)
$$

и для любой точки $z \in \mathscr{B}_{i}$ имеет место свойство: $\overline{W^{u}(z)}=\mathscr{B}_{i}$. Положим $g=f^{k}$.

Из доказательства леммы 1 следует, что $\operatorname{dim} W^{u}(z)=1$ для любой точки $z \in \mathscr{B}_{i}$, и $W^{u}(z)$ принадлежит $\mathscr{B}_{i}$.

Обозначим $F_{i}^{u}$ семейство неустойчивых многообразий $W^{u}(z)$ для всех точек $z \in \mathscr{B}_{i}$.

Лемма 2. Пусть $f: M^{3} \rightarrow M^{3}$ является $A$-диффеоморфизмом, неблуждаюиее мнохество которого содержит двумерный аттрактор $\mathscr{B}$ с носителем $M_{\mathscr{B}}^{2}$. Tогда

1) $\mathscr{B}=M_{\mathscr{B}}^{2}$;

2) семейство $F_{i}^{u}$ является непрерывным слоением без особенностей, заданнымм на $M_{i}^{2}$

3) $M_{\mathscr{B}}^{2}$ является облединением $M_{1}^{2} \cup \cdots \cup M_{k}^{2}$ непересекаюшихся поверхностей таких, что каждая из них гомеоморфна 2-тору $T^{2}$ и $\mathscr{B}_{i}=M_{i}^{2}$.

ДОКАЗАТЕЛЬСТВО. Положим $g=f^{k}$.

Очевидно, что для любых $i \in\{1, \ldots, k\}$ существует поверхность $M_{i}^{2} \subseteq M^{2}$, которая является носителем для базисного множества $\mathscr{B}_{i}$ диффеоморфизма $g$. Покажем, что $\mathscr{B}_{i}=M_{i}^{2}$.

Так как по предположению $\operatorname{dim} \mathscr{B}_{i}=2$ и $\mathscr{B}_{i} \subset M_{i}^{2}$, то $\mathscr{B}_{i}$ содержит непустое открытое (во внутренней топологии $M_{i}^{2}$ ) множество, скажем $V$ (см. теорему 4.3 из [23]). 
Пусть $z$ - любая точка, принадлежащая int $V$. Согласно [8] существует $\alpha>0$ такое, что у точки $z$ есть замкнутая окрестность $U_{z} \subset V$, гомеоморфная прямому произведению $\check{W}_{\alpha}^{s}(z) \times W_{\alpha}^{u}(z)$, где $\check{W}_{\alpha}^{s}(z)=\mathscr{B} \cap W_{\alpha}^{s}(z)$ и $W_{\alpha}^{s}(z), W_{\alpha}^{u}(z)$ - замкнутые $\alpha$-окрестности точки $z$ в некоторой внутренней метрике многообразий $W^{s}(z), W^{u}(z)$ соответственно. Это значит, что для любой точки $w \in U_{z}$ существует единственная пара точек

$$
w^{s} \in \check{W}_{\alpha}^{s}(z), \quad w^{u} \in W_{\alpha}^{u}(z) \quad \text { таких, что } w=W^{u}\left(w^{s}\right) \cap W_{\alpha}^{s}\left(w_{u}\right)
$$

Определим проекцию $\pi_{z}: U_{z} \rightarrow W^{s}(z)$ следующим образом. Для $w \in U_{z}$ положим $\pi_{z}(w)=w^{s}$.

Так как $V$ - открытое подмножество поверхности $M^{2}$, топологически вложенной в $M^{3}$, то можно предположить без потери общности, что $U_{z}$ гомеоморфно замкнутому 2-диску.

Положим $U_{z}^{s}=\pi_{z}\left(U_{z}\right)$. Покажем, что существует простой замкнутьй путь $\gamma \subset U_{z}^{s}$ такой, что $z \in \operatorname{int} \gamma$.

Хорошо известно (см., например, [24]), что любая замкнутая кривая является ручной на диске. Таким образом, существует открытая окрестность $D_{z} \subset U_{z}$ точки $z$ такая, что

1) множество $W^{u}(z) \cap D_{z}$ состоит в точности из одной связной компоненты, скажем $\lambda \subset W^{u}(z)$

2) множество $D \backslash \lambda$ состоит из двух связных компонент, скажем $D_{1}, D_{2}$;

3) $\pi\left(D_{1}\right) \cap \pi\left(D_{2}\right)=\varnothing$.

Выберем любые точки

$$
z_{1} \in \pi\left(D_{1}\right), \quad z_{2} \in \pi\left(D_{2}\right) .
$$

Так как множества $D_{1} \cup \lambda, D_{2} \cup \lambda$ являются линейно-связными подмножествами и $\pi$ - непрерьвное отображение, то $\pi\left(D_{1} \cup \lambda\right), \pi\left(D_{2} \cup \lambda\right)$ - также линейно-связные подмножества. Кроме того, так как $\pi\left(D_{1} \cup \lambda\right), \pi\left(D_{2} \cup \lambda\right)$ являются хаусдорфовыми подпространствами (в индуцированной топологии), то существуют ${ }^{2}$ простая дуга $\gamma_{1} \subset \pi\left(D_{1} \cup \lambda\right)$, соединяющая точки $z_{1}, z$, и простая дуга $\gamma_{2} \subset \pi\left(D_{2} \cup \lambda\right)$, соединяющая точки $z_{2}, z$. Тогда дуга $\gamma=\gamma_{1} \cup \gamma_{2}$ является простой дугой, соединяющей точки $z_{1}$ и $z_{2}$. По построению $z \in \operatorname{int} \gamma$.

Введем параметр $t \in[-1,1]$ на дуге $\gamma$ такой, что $\gamma(-1)=z_{1}, \gamma(0)=z$ и $\gamma(1)=z_{2}$. Для любого $t \in[-1,1]$ определим $l_{t}=W_{\alpha}^{u}(\gamma(t))$ и положим

$$
F_{z}^{u}=\bigcup_{t \in[-1,1]} l_{t}
$$

Для любой точки $\gamma(t)$ неустойчивое многообразие $W_{\alpha}^{u}(\gamma(t))$ трансверсально пересекает устойчивое многообразие $W_{\alpha}^{s}(z)$ в единственной точке.

\footnotetext{
${ }^{2}$ См., например, [25, предложение $\left.6.3 .12(\mathrm{a})\right]$, которое утверждает, что хаусдорфово пространство линейно связно тогда и только тогда, когда для любых точек $x_{1}, x_{2}\left(x_{1} \neq x_{2}\right)$ существует гомеоморфное вложение $h: I \rightarrow X$ замкнутого единичного интервала $I=[0,1]$ в пространство $X$, удовлетворяющее условиям $h(0)=x_{1}, h(1)=x_{2}$ (т.е. $X$ является дугообразно связным).
} 
Пусть $V_{0}(z)$ - диск, состоящий из всех точек, принадлежаших объединению кривых $l_{t}, t \in[-1,1]$. Согласно теореме о непрерьвной зависимости неустойчивых многообразий от начальных условий (см., например, [8]) семейство кривых $F_{z}^{u}$ локально гомеоморфно семейству прямых, параллельных оси $O x$ на евклидовой плоскости, посредством некоторого гомеоморфизма $h_{z}: V_{0}(z) \rightarrow \mathbb{R}^{2}$. Следовательно, $F_{z}^{u}$ - непрерывное слоение, заданное на замкнутом диске $V_{0}(z)$.

Пусть $y \in \mathscr{B}_{i}$ - любая точка. Так как $\overline{W^{u}(y)}=\mathscr{B}_{i}$, то существует точка $w \in$ int $\gamma$ такая, что $[y, w]^{u} \subset W^{u}(y)$. Согласно теореме о непрерывной зависимости неустойчивых многообразий от начальных условий существуют открытые окрестности $U_{w} \subset$ int $\gamma$ точки $w$ и $U_{y} \subset M_{i}^{2}$ точки $y$ такие, что для любой точки $y^{\prime} \in U_{y}$ существует $w^{\prime} \in U_{w}$ такая, что $\left[w^{\prime}, y^{\prime}\right]^{u} \subset W^{u}\left(w^{\prime}\right)$. Следовательно, точка $y^{\prime}$ принадлежит $\mathscr{B}_{i}$. Это означает, что множество $\mathscr{B}_{i}$ открыто. Так как $\mathscr{B}_{i}$ замкнуто, то оно совпадает с поверхностью $M_{i}^{2}$. Так как $\mathscr{B}_{i} \cap \mathscr{B}_{j}=\varnothing$ для $i \neq j$, то $M_{i}^{2} \cap M_{j}^{2}=\varnothing$.

Таким образом, $\mathscr{B}=M_{\mathscr{B}}^{2}$ и $M_{\mathscr{B}}^{2}$ является объединением $M_{1}^{2} \cup \cdots \cup M_{k}^{2}$ непересекающихся поверхностей.

Из приведенных вьше рассуждений следует, что для любой точки $b \in \mathscr{B}_{i}$ существует окрестность $U_{b}$ и гомеоморфизм $h_{b}: U_{b} \rightarrow \mathbb{R}^{2}$ такой, что $h_{b}$ отображает пересечения кривых из $F_{i}^{u}$ с окрестностью $U_{b}$ на семейство прямых, параллельных оси $O x$. Таким образом, семейство $F_{i}^{u}$ является транзитивным слоением без особенностей, заданным на $M_{i}^{2}$. Следовательно, поверхность $M_{i}^{2}$ гомеоморфна либо бутылке Клейна, либо тору. В силу [26] любое слоение без особенностей на бутылке Клейна должно иметь, по крайней мере, один замкнутый слой. Как следствие получаем, что бутылка Клейна не допускает транзитивных слоений. Таким образом, многообразие $M_{i}^{2}$ гомеоморфно тору, и лемма полностью доказана.

ЛЕмма 3. Поверхность $M_{i}^{2}$ является ручной.

ДокАЗАТЕЛьСтво. Из доказательства леммы 2 следует, что для любой точки $z \in M_{i}^{2}$ существует окрестность $V_{0}(z) \subset M_{i}^{2}$ такая, что

1) $V_{0}(z)$ гомеоморфна прямому произведению $\gamma \times W_{\alpha}^{u}(z)$, где $\gamma$ - простая кривая, принадлежащая $W_{\alpha}^{s}(z)$;

2) $V_{0}(z)$ есть объединение гладких кривых $W_{\alpha}^{u}(w), w \in \gamma$, которые принадлежат слоям слоения $F_{i}^{u}$ и образуют слоение $\widetilde{F}^{u}$ на окрестности $V_{0}(z)$;

3 ) любая кривая $W_{\alpha}^{u}(w)$ пересекает $W_{\alpha}^{s}(z)$ точно в одной точке;

4) кривая $\gamma$ является локальной секущей (в топологическом смысле) для слоения $\widetilde{F}^{u 3}$.

Покажем, что существует окрестность $B_{z}$ точки $z$, гомеоморфная 3-диску, и вложение $h_{z}: B_{z} \rightarrow \mathbb{R}^{3}$ такое, что $h_{z}\left(B_{z} \cap V_{0}(z)\right)=D_{0}$.

Без потери обшности мы можем предположить, что существует окрестность $\widetilde{B}_{z}$ точки $z$, которая гомеоморфна 3-диску, такая, что

1) $W_{\alpha}^{s}(z) \subset \widetilde{B}_{z}, V_{0}(z) \subset \widetilde{B}_{z}$;

\footnotetext{
${ }^{3}$ Говорят, что $\gamma$ есть локальная секущая (в топологическом смысле) для слоения $\widetilde{F}^{u}$, если для любой точки $d \in \gamma$ существует окрестность $V_{d} \subset V_{0}(z)$ такая, что для любого слоя $l \subset \widetilde{F}^{u}$ такого, что $\left(l \cap V_{d}\right) \neq \varnothing$, пересечение $\left(l \cap V_{d}\right) \cap \gamma$ состоит в точности из одной точки $x_{l}$ и $V_{d} \backslash \gamma$ состоит из двух компонент связности, каждая из которых содержит в точности одну компоненту множества $l \backslash\left\{x_{l}\right\}$.
} 
2) существует диффеоморфизм $g:{\widetilde{\widetilde{B}_{z}}}_{\boldsymbol{1}} \rightarrow B_{0}^{3}$ такой, что $g\left(W_{\alpha}^{s}(z)\right) \subset O x y, g(z)=$ $O(0,0,0)$, где $B_{0}^{3}$ - замкнутьй единичный шар в $\mathbb{R}^{3}$.

Положим

$$
V=g\left(V_{0}(z)\right), \quad Q=g\left(W_{\alpha}^{s}(z)\right)
$$

и обозначим через $\widehat{F}^{u}$ слоение, заданное на $V$, являющееся образом слоения $\widetilde{F}^{u}$ при отображении $g$. По построению кривая $\lambda=g(\gamma)$ является локальной секущей (в топологическом смысле) для слоения $\widehat{F}^{u}$. Выберем замкнутую простую дугу $\lambda_{1} \subset$ int $\lambda$, обозначим через $a, b$ концевые точки дуги $\lambda_{1}$. Обозначим через $a_{0}\left(b_{0}\right)$ точку, принадлежащую компоненте связности множества $\lambda \backslash$ int $\lambda_{1}$, для которой точка $a(b)$ принадлежит ее границе. Обозначим через $\gamma_{a_{0} a}$ и $\gamma_{b b_{0}}$ простые замкнутые дуги, принадлежашие $\lambda$, для которых точки $a_{0}, a$ и $b, b_{0}$ являются соответственно концевьми точками.

Покажем, что существуют простые кусочно-линейные дуги $\tilde{\gamma}_{a_{0} a}$ и $\tilde{\gamma}_{b b_{0}}$ с конщевыми точками $a_{0}, a$ и $b, b_{0}$ соответственно, принадлежащие $Q$ и удовлетворяющие следующим условиям:

1) $\tilde{\gamma}_{a_{0} a} \cap \tilde{\gamma}_{b b_{0}}=\varnothing$

2) $\tilde{\gamma}_{a_{0} a} \cap \lambda_{1}=a, \tilde{\gamma}_{b b_{0}} \cap \lambda_{1}=b$;

$3)$ концевые точки каждого линейного звена кривой $\tilde{\gamma}_{a_{0} a}\left(\right.$ соответственно $\left.\tilde{\gamma}_{b b_{0}}\right)$ принадлежит кривой $\gamma_{a_{0} a}$ (соответственно $\gamma_{b b_{0}}$ ).

Покажем существование кривой $\gamma_{a_{0}} a$ (существование кривой $\gamma_{b b_{0}}$ устанавливается аналогично). Положим $\nu=\gamma_{a_{0}} \backslash\{a\}$ и выберем открытую окрестность $U_{\nu} \subset V$ множества $\nu$ такую, что

1) $U_{\nu} \cap\left(\lambda_{1} \cup \gamma_{b b_{0}}\right)=\varnothing$

2) $U_{\nu}$ допускает триангуляцию $\Sigma=\bigcup_{i \in \mathbb{Z}^{+}} \sigma_{i}$ такую, что для любой точки $x \in U_{\nu}$ любая окрестность $U_{x} \subset U_{\nu}$ точки $x$ пересекает только конечное число симплексов из объединения $\bigcup_{i \in \mathbb{Z}^{+}} \sigma_{i}$.

Введем параметр $t \in[0, \infty)$ на дуге $\nu$ такой, что $\nu(0)=a_{0}$ и $\nu(t)$ стремится к $a$ при $t \in+\infty$.

Так как $\nu(t)$ стремится к $a$ (при $t \in+\infty)$, то существует последовательность чисел

$$
0=t_{i_{0}}<t_{i_{1}}<\cdots<t_{i_{k}}<\cdots, \quad t_{i_{k}} \rightarrow \infty \text { при } k \rightarrow+\infty
$$

такая, что

$$
\nu\left(t_{i_{k}}\right) \in \sigma_{i_{k}}, \quad \sigma_{i_{j}} \cap \sigma_{i_{j+1}} \neq \varnothing, \quad \text { int } \sigma_{i_{j}} \cap \operatorname{int} \sigma_{i_{j+1}}=\varnothing
$$

и для любого $t>t_{i_{k}}$ точка $\nu(t)$ не принадлежит $\bigcup_{j \leqslant k} \sigma_{i_{j}}$. Обозначим $l_{k}$ отрезок прямой, соединяющей точки $\nu\left(t_{i_{k}}\right)$ и $\nu\left(t_{i_{k+1}}\right), k \in \mathbb{Z}^{+}$. По построению последовательность точек $\left\{\nu\left(t_{i_{k}}\right)\right\}$ стремится к $a$ при $k \rightarrow+\infty$. Тогда множество $\tilde{\gamma}_{a_{0} a}=\bigcup_{i \in \mathbb{Z}^{+}} l_{i} \cup\{a\}$ будет искомой кривой.

Без потери общности мы можем предположить, что существует число $N>0$ такое, что для любого $c \in[-N, N]$ плоскость $P_{c}$, заданная уравнением $z=c$, пересекает любой слой слоения $\widehat{F}^{u}$ в точности в одной точке.

Для любой точки $x \in \lambda$ обозначим через $\widetilde{L}_{x}^{u}$ замкнутую дугу такую, что

1) $\widetilde{L}_{x} \subset L_{x}^{u}$, где $L_{x}^{u}$ есть слой слоения $\widehat{F}^{u}$, проходящий через точку $x$;

2) $\widetilde{L}_{x}$ лежит между плоскостями $P_{-N}: z=-N, P_{N}: z=N$. 
Тогда дуга $\widetilde{L}_{\nu\left(t_{i_{k}}\right)}^{u}$ может быть задана уравнениями

$$
x=x_{k}(z), \quad y=y_{k}(z), \quad z=z, \quad z \in[-N, N] .
$$

Заметим, что по построению точка $\nu\left(t_{i_{k}}\right)$ имеет координаты $\left(x_{k}(0), y_{k}(0), 0\right)$.

Обозначим через $S_{k}$ диск, определенный следующими уравнениями:

$$
\begin{gathered}
x=x_{k}(z)+s_{k}\left(x_{k+1}(z)-x_{k}(z)\right), \quad y=y_{k}(z)+s_{k}\left(y_{k+1}(z)-y_{k}(z)\right), \\
z=z, \quad z \in[-N, N], \quad s_{k} \in[0,1] .
\end{gathered}
$$

Положим

$$
S_{a_{0} a}=\bigcup_{k \in \mathbb{Z}^{+}} S_{k} \cup \widetilde{L}_{a}^{u}
$$

По конструкции $S_{a_{0}}$ является кусочно-гладким диском. Используя кусочно-линейную кривую $\tilde{\gamma}_{b b_{0}}$ построим кусочно-гладкий диск $S_{b b_{0}}$, аналогичный $S_{a_{0} a}$.

Положим

$$
S_{a b}=\bigcup_{x \in \lambda_{1}} \tilde{L}_{x}^{u}, \quad S=S_{a_{0} a} \cup S_{b b_{0}} \cup S_{a b}, \quad \nu_{-N}=S \cap P_{-N}, \quad \nu_{N}=S \cap P_{N} .
$$

Нетрудно найти гладко вложенные диски $S_{1}, S_{2}$ такие, что

1) $S_{1}, S_{2}$ трансверсально пересекают каждую плоскость $P_{c}, c \in[-N, N]$;

2) int $S_{i} \cap \operatorname{int} S=\varnothing, S_{i} \cap S=\widetilde{L}_{a_{0}}^{u} \cup \widetilde{L}_{b_{0}}^{u}, i=1,2$;

$3) \operatorname{int} S_{1} \cap \operatorname{int} S_{2}=\varnothing, S_{1} \cap S_{2}=\widetilde{L}_{a_{0}}^{u} \cup \widetilde{L}_{b_{0}}^{u}$;

4) граница диска $S_{i}$ состоит из кривьг $\widetilde{L}_{a_{0}}^{u}, \widetilde{L}_{b_{0}}^{u}$ и кривых $\nu_{-N}^{i}=S_{i} \cap P_{-N}, \nu_{N}^{i}=$ $S_{i} \cap P_{N}, i=1,2$.

5) кривые $\nu_{-N}^{1}, \nu_{-N}^{2}$ (соответственно $\left.\nu_{N}^{1}, \nu_{N}^{2}\right)$ образуют границу замкнутого диска $D_{-N} \subset P_{-N}$ (соответственно $\left.D_{N} \subset P_{N}\right)$, который содержит кривую $\nu_{-N}$ (соответственно $\left.\nu_{N}\right)$, делящую диск $D_{-N}\left(\right.$ соответственно $\left.D_{N}\right)$ на два диска: диск $D_{-N}^{1}$, ограниченный кривыми $\nu_{-N}, \nu_{-N}^{1}$ и диск $D_{-N}^{2}$, ограниченньй кривыми $\nu_{-N} \nu_{-N}^{2}\left(\right.$ соответственно диск $D_{N}^{1}$ ограниченньй кривыми $\nu_{N}, \nu_{N}^{1}$ и диск $D_{N}^{2}$, ограниченный кривыми $\left.\nu_{N} \nu_{N}^{2}\right)$.

Обозначим через $B_{i}$ замкнутое множество, ограниченное объединением $S \cup S_{i} \cup$ $D_{-N}^{i} \cup D_{N}^{i}$.

Так как пересечение множеств $B_{i} \cap P_{c}$ гомеоморфно стандартному диску $D_{0}$, то $B_{i}$ гомеоморфно $D_{0} \times[-N, N]$ и, следовательно, $B_{i}$ гомеоморфно стандартному шару

$$
B_{0}=\left\{(x, y, z) \in \mathbb{R}^{3} \mid x^{2}+y^{2}+z^{2} \leqslant 1\right\} .
$$

Тогда существует окрестность $U_{S}$ диска $S$ такая, что $U_{S} \cap B_{1} \quad$ (соответственно $\left.U_{S} \cap B_{2}\right)$ гомеоморфна $D_{0} \times[-1,0]$ (соответственно $\left.D_{0} \times[0,1]\right)$. Таким образом сушествует окрестность $U_{O}$ точки $O$ и гомеоморфизм $h_{O}: \overline{U_{O}} \rightarrow \mathbb{R}^{3}$ такой, что $h_{O}\left(U_{O} \cap S\right)$ является стандартным замкнутьм диском $D_{0}$.

Множество $B_{z}=g^{-1}\left(U_{O}\right)$ является окрестностью точки $z$ в $M^{3}$ и отображение $h_{z}=h_{O} \circ g: B_{z} \rightarrow \mathbb{R}^{3}$ удовлетворяет следующему условию: $h_{z}\left(B_{z} \cap V_{0}(z)\right)$ является стандартным замкнутьм диском $D_{0}$. Это означает, что поверхность $M_{i}^{2}$ ручная. Лемма доказана. 
3. Доказательство теоремы 2. Доказательство теоремы 2 следует из лемм 4 и 5 , которые будут доказаны ниже.

Для любой точки $x \in M_{i}^{2}$ положим

$$
L_{i}^{s}(x)=W^{s}(x) \cap M_{i}^{2}
$$

и обозначим

$$
F_{i}^{s}=\bigcup_{x \in M_{i}^{2}} L_{i}^{s}(x) .
$$

Из локальной структуры прямого произведения и доказательства леммы 2 следует, что существует $\alpha>0$ такое, что для любой точки $z \in M_{i}^{2}$ существует окрестность $V_{0}(z)$ такая, что

1) $W_{\alpha}^{u}(z) \subset V_{0}(z)$ и для любой точки $y \in W_{\alpha}^{u}(z)$ пересечение $W_{\alpha}^{s}(y) \cap V_{0}(z)$ является простой кривой $\gamma_{y}^{s}$;

2) семейство кривых $\bigcup_{y \in W_{\alpha}^{u}(z)} \gamma_{y}^{s}$ является непрерьвным слоением на окрестности $V_{0}(z)$, т.е. существует гомеоморфизм $q_{z}: V_{0}(z) \rightarrow \mathbb{R}^{2}$, отображающий семейство $\bigcup_{y \in W_{\alpha}^{u}(z)} \gamma_{y}^{s}$ в семейство прямых линий, параллельных оси $O x$ на плоскости $\mathbb{R}^{2}$;

3) любая кривая $\gamma_{y}^{s}$ является локальной трансверсалью (в топологическом смысле) к слоению $F_{i}^{u}$.

Так как $\mathscr{B}_{i}=M_{i}^{2}$ и для любой точки $x \in \mathscr{B}_{i}$ пересечение $W^{s}(x) \cap \mathscr{B}_{i}$ плотно в $\mathscr{B}_{i}$, то любой слой слоения $F_{i}^{s}$ плотен в $M_{i}^{2}$. Таким образом, $F_{i}^{s}$ является транзитивньм слоением без особенностей на торе $M_{i}^{2}$.

Представим тор $M_{i}^{2}$ как фактор-пространство $\mathbb{R}^{2} / \Gamma$, где $Г$ - дискретная группа движений $\gamma_{m, n}$ на плоскости $\mathbb{R}^{2}$, заданных формулами

$$
\gamma_{m, n}: \quad \bar{x}=x+m, \quad \bar{y}=y+n, \quad m, n \in \mathbb{Z}
$$

Обозначим через $\pi: \mathbb{R}^{2} \rightarrow M_{i}^{2}$ естественную проекцию и через $g_{*}$ - автоморфизм группы $\Gamma$, индуцированньй диффеоморфизмом $g\left(g=f^{k}\right)$. Заметим, что автоморфизм $g_{*}$ можно задать следующим образом. Пусть $\bar{g}: \mathbb{R}^{2} \rightarrow \mathbb{R}^{2}$ - накрывающий диффеоморфизм для $g: M_{i}^{2} \rightarrow M_{i}^{2}$, т.е. $\pi \circ \bar{g}=g \circ \pi$, и предположим, что $\bar{g}$ задан формулами

$$
\bar{g}: \quad \bar{x}=g_{1}(x, y), \quad \bar{y}=g_{2}(x, y) .
$$

Тогда для любого $\gamma_{m, n} \in \Gamma$ положим

$$
g_{*}\left(\gamma_{m, n}\right)=\gamma_{m^{\prime}, n^{\prime}}, \quad \text { где } \quad m^{\prime}=g_{1}(m, n)-g_{1}(0,0), \quad n^{\prime}=g_{2}(m, n)-g_{2}(0,0) \text {. }
$$

Лемма 4. Автоморфизм $g_{*}$ является гиперболическим, т.е. собственные значения $\lambda_{1}, \lambda_{2}$ матриив

$$
\mathbf{A}=\left(\begin{array}{ll}
a & b \\
c & d
\end{array}\right) \in S L(2, \mathbb{Z})
$$

задающей автоморфизм $g_{*}$, удовлетворяют условию $\left|\lambda_{1}\right|<1,\left|\lambda_{2}\right|>1$. 
ДокАЗАТЕльСтво. Слоения $F_{i}^{u}$ и $F_{i}^{s}$ транзитивны и трансверсальны на торе $M_{i}^{2}$. Это означает, что они образуют транзитивную 2-ткань на $M_{i}^{2}$. Тогда согласно $[27$, теорема 1], существуют различные иррациональные числа $\mu^{u}, \mu^{s}$ (которые являются числами вращения Пуанкаре слоений $F_{i}^{u}$ и $F_{i}^{s}$ соответственно) и гомеоморфизм $\varphi: M_{i}^{2} \rightarrow$ $M_{i}^{2}$ такой, что $\varphi$ отображает слоения $F_{i}^{u}$ и $F_{i}^{s}$ в линейные слоения $L_{\mu}^{u}$ и $L_{\mu}$ соответственно (слоение $L_{\mu}$ есть образ при проекции $\pi$ слоения $\bar{L}_{\mu^{\sigma}}$, каждьй слой которого задается уравнением $\left.y=\mu^{\sigma} x+c, \sigma \in\{u, s\}, c \in \mathbb{R}^{1}\right)$.

Обозначим через $\bar{F}_{i}^{\sigma}$ слоениена $\mathbb{R}^{2}$, которое является накрьваюшим для слоения $F_{i}^{\sigma}$, $\sigma \in\{s, u\}$. Тогда имеется накрьвающий гомеоморфизм $\bar{\varphi}: \mathbb{R}^{2} \rightarrow \mathbb{R}^{2}$ для гомеоморфизма $\varphi$, отображаюший слоения $\bar{F}_{i}^{u}$ и $\bar{F}_{i}^{s}$ в линейные слоения $\bar{L}_{\mu} u$ и $\bar{L}_{\mu^{s}}$ соответственно. Отсюда следует, что любой слой $l^{u}$ слоения $\bar{F}_{i}^{u}$ пересекает любой слой $l^{s}$ слоения $\bar{F}_{i}^{s}$ в точности в одной точке.

Покажем теперь, что автоморфизм $g_{*}$ является гиперболическим. Предположим противное. Пусть $p \in \operatorname{Per}(g)$ - периодическая точка периода $m \geqslant 1$. Без ограничения общности можно предположить, что $p=\pi(O)$ (где $O$ - начало евклидовой системы координат на плоскости). Обозначим через $\bar{g}_{m}$ накрьвающий гомеоморфизм для $g^{m}$ такой, что $\bar{g}_{m}(O)=O$. Множество $\mathscr{O}=\bigcup_{\gamma \in \Gamma} \gamma(O)$ есть решетка на плоскости $\mathbb{R}^{2}$. Матрица $\mathbf{A}^{m}$ определяет автоморфизм $g_{*}^{m}$. Обозначим $\mathscr{A}_{m}: \mathbb{R}^{2} \rightarrow \mathbb{R}^{2}$ линейное отображение, определенное матрицей $\mathbf{A}^{m}$. Из определения $g_{*}$ следует, что $\left.\bar{g}_{m}\right|_{\mathscr{O}}=\left.\mathscr{A}_{m}\right|_{\mathscr{O}}$. Так как модуль собственных значений матрицы $A^{m}$ равен 1 , то имеется периодическая точка $O_{1} \in \mathscr{O}\left(O_{1} \neq O\right)$ некоторого периода $l \geqslant 1$ отображения $\left.\mathscr{A}_{m}\right|_{\mathscr{O}}$. Следовательно, $O_{1}$ есть периодическая точка периода $l$ диффеоморфизма $\bar{g}_{m}$. Тогда точки $O, O_{1}$ являются неподвижными точками диффеоморфизма $\bar{g}_{m}^{l}$. Обозначим через $l_{O}^{u}$ (соответственно $l_{O_{1}}^{s}$ ) слой слоения $\bar{F}_{i}^{u}$ (соответственно $\bar{F}_{i}^{s}$ ), проходящий через точку $O$ (соответственно $\left.O_{1}\right)$. Так как $l_{O}^{u} \cap l_{O_{1}}^{s} \neq \varnothing$ и $l_{O}^{u}, l_{O_{1}}^{s}$ являются инвариантными неустойчивым и устойчивьм многообразиями неподвижных седловых (в топологическом смысле) точек $O, O_{1}$ соответственно, то имеется бесконечное множество гетероклинических точек диффеоморфизма $\bar{g}_{m}^{l}$, принадлежащих пересечению $l_{O}^{u} \cap l_{O_{1}}^{s}$. Получаем противоречие с тем, что $l_{O}^{u} \cap l_{O_{1}}^{s}$ состоит в точности из одной точки. Лемма доказана.

Обозначим $\mathscr{G}$ линейный автоморфизм тора $M_{i}^{2}$ такой, что $\mathscr{G}_{*}=g_{*}$.

Согласно [1, предложение 2.1] существует непрерывное гомотопное тождественному отображение $h: M_{i}^{2} \rightarrow M_{i}^{2}$ такое, что $\mathscr{G} h=h g$.

Лемма 5. Отображсение $h$ есть гомеоморфизм.

ДокАЗАтЕльство. Пусть $\bar{h}: \mathbb{R}^{2} \rightarrow \mathbb{R}^{2}$ - накрьвающее отображение для $h$. Разделим доказательство леммы на три шага.

Шаг 1. Покажем, что если точки $\bar{x}, \bar{y} \in \mathbb{R}^{2}, \bar{x} \neq \bar{y}$, принадлежат одному и тому же слою $l^{\sigma}$ слоения $\bar{F}_{i}^{\sigma}$, то $\bar{h}(\bar{x}) \neq \bar{h}(\bar{y}), \sigma \in\{s, u\}$.

Рассмотрим для определенности случай $\sigma=u$ (для $\sigma=s$ доказательство аналогично) и предположим противное. Пусть имеется слой $l^{u}$ слоения $\bar{F}_{i}^{u}$ и точки $\bar{x}, \bar{y} \in l^{u}$ такие, что $\bar{h}(\bar{x})=\bar{h}(\bar{y})$. Положим $x=\pi(\bar{x}), y=\pi(\bar{y}), L^{u}=\pi\left(l^{u}\right)$ и обозначим через $[x, y]^{u} \subset L^{u}$ замкнутую дугу с концевыми точками $x, y$. Пусть $p$ - любая периодическая точка некоторого периода $l \geqslant 1$ ограничения диффеоморфизма $g$ на $M_{i}^{2}$. Обозначим $L_{p}^{s}$ слой слоения $F_{i}^{s}$, проходящий через точку $p$. Мы имеем две возможности:

а) точка $p$ принадлежит $[x, y]^{u}$; 
b) точка $p$ не принадлежит $[x, y]^{u}$.

Поскольку слой $L_{p}^{s}$ плотен на поверхности $M_{i}^{2}$, то в случае b) имеется точка $v \in L_{p}^{s} \cap$ $(x, y)^{u}$.

Следовательно, имеются два случая:

$\bar{a})$ существует точка $\bar{p} \in \pi^{-1}(p)$, принадлежащая дуге $[\bar{x}, \bar{y}]^{u} \subset l^{u}$;

$\overline{\mathrm{b}})$ имеются точки $\bar{p} \in \pi^{-1}(p), \bar{v} \in \pi^{-1}(v)$ такие, что $\bar{p}$ и $\bar{v}$ принадлежат одному и тому же слою слоения $\bar{F}^{s}$ и точка $\bar{v}$ принадлежит дуге $(\bar{x}, \bar{y})^{u} \subset l^{u}$.

Рассмотрим поднятие $\bar{g}_{l}$ диффеоморфизма $g^{l}$ такое, что $\bar{g}_{l}(\bar{p})=\bar{p}$. Тогда в обоих случаях $\overline{\mathrm{a}})$ и $\overline{\mathrm{b}}$ ) мы имеем

$$
\rho\left(\bar{g}_{l}^{n}(\bar{x}), \bar{g}_{l}^{n}(\bar{y})\right) \rightarrow+\infty \quad \text { при } n \rightarrow+\infty .
$$

Заметим, что согласно $[1$, лемма 3.4$]$ отображение $\bar{h}$ является собственным ${ }^{4}$. Следовательно, согласно [28] имеется число $r>0$ такое, что для любых точек $\bar{x}_{1}, \bar{x}_{2} \in \mathbb{R}^{2}$, удовлетворяющих условию $\bar{h}\left(\bar{x}_{1}\right)=\bar{h}\left(\bar{x}_{2}\right)$, имеет место ${ }^{5}$ неравенство $\rho\left(\bar{x}_{1}, \bar{x}_{2}\right)<r$. Так как $\mathscr{G}^{l} \circ h=h \circ g^{l}$ и $h$ гомотопен тождественному, то существует накрьвающее отображение $\overline{\mathscr{G}}_{l}$ для линейного диффеоморфизма $\mathscr{G}^{l}$ такое, что $\overline{\mathscr{G}}_{l} \circ \bar{h}=\bar{h} \circ g_{l}$. Тогда для любого $n \in \mathbb{Z}$ имеем $\bar{h}\left(\bar{g}^{n}(\bar{x})\right)=\overline{\mathscr{G}}_{l}^{n}(\bar{h}(\bar{x}))=\overline{\mathscr{G}}_{l}^{n}(\bar{h}(\bar{y}))=\bar{h}\left(\bar{g}^{n}(\bar{y})\right)$. Следовательно, $\rho\left(\bar{g}^{n}(\bar{x}), \bar{g}^{n}(\bar{y})\right)<r$. Получаем противоречие с тем, что $\rho\left(\bar{g}_{l}^{n}(\bar{x}), \bar{g}_{l}^{n}(\bar{y})\right) \rightarrow+\infty$ при $n \rightarrow+\infty$.

Шаг 2. Так как $\mathscr{G}$ - линейньй гиперболический автоморфизм тора, то через каждую точку $x \in M_{i}^{2}$ проходят два трансверсальных многообразия: устойчивое $W^{s}(x)$ и неустойчивое $W^{u}(x)$, которые являются образами проекции $\pi: \mathbb{R}^{2} \rightarrow M_{i}^{2}$ прямых линий $w^{s}(\bar{x}), w^{s}(\bar{y})$ соответственно, проходящих через любую точку $\bar{x} \in \pi^{-1}(x)$.

Покажем, что для любой точки $\bar{x} \in \mathbb{R}^{2}$ справедливо свойство:

$$
\bar{h}\left(l_{\bar{x}}^{\sigma}\right)=w^{\sigma}(\bar{h}(\bar{x}))
$$

где $l_{\bar{x}}^{\sigma}-$ слой слоения $\bar{F}_{i}^{\sigma}$.

Рассмотрим для определенности случай $\sigma=s$ (в случае $\sigma=u$ доказательство аналогично). Сначала покажем, что $\bar{h}\left(l_{\bar{x}}^{\sigma}\right) \subset W^{\sigma}(\bar{h}(\bar{x}))$. Пусть $\bar{y}$ - любая точка, принадлежащая $l_{\bar{x}}^{\sigma}(\bar{y} \neq \bar{x})$. Положим $x=\pi(\bar{x}), y=\pi(\bar{y})$. Так как $\lim _{n \rightarrow+\infty} d\left(g^{n}(x), g^{n}(y)\right) \rightarrow 0$ $\left(d\right.$ - метрика на торе $\left.M_{i}^{2}\right)$, то из непрерьвности отображения $h$ следует, что

$$
\lim _{n \rightarrow+\infty} d\left(h\left(g^{n}(x)\right), h\left(g^{n}(y)\right)\right)=\lim _{n \rightarrow+\infty} d\left(\mathscr{G}^{n}(h(x)), \mathscr{G}^{n}(h(y))\right)=0 .
$$

Отсюда следует, что $h(y) \subset W^{s}(h(x))$. Так как $\bar{h}$ является накрывающим отображением для $h$, то $\bar{h}\left(l_{\bar{x}}^{s}\right) \subset w^{s}(\bar{h}(\bar{x}))$.

\footnotetext{
${ }^{4}$ Отображение $\bar{h}$ назьвается собственным, если прообраз компактного множества является ограниченным множеством.

${ }^{5}$ Для удобства читателя мы повторяем здесь рассуждение из [28]. Действительно, поскольку отображение $\bar{h}$ является собственным, то имеется $r>0$ такое, что прообраз фундаментальной области П группы $Г$ принадлежит открытому диску $D_{r / 2}=\left\{(x, y) \in \mathbb{R} \mid x^{2}+y^{2}<r^{2} / 4\right\}$. Пусть $\gamma \in \Gamma$ такой, что $\gamma\left(\bar{h}\left(\bar{x}_{1}\right)\right) \in \Pi$. Так как отображение $h$ гомотопно тождественному и

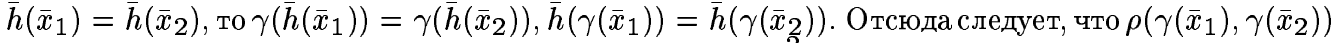
$<r$. Поскольку отображение $\gamma$ является изометрией на $\mathbb{R}^{2}$, то $\rho\left(\bar{x}_{1}, \bar{x}_{2}\right)<r$.
} 
Покажем теперь, что $\bar{h}\left(l_{\bar{x}}^{s}\right)=w^{s}(\bar{h}(\bar{x}))$. Предположим противное. Так как $\bar{h}\left(w^{s}(\bar{x})\right)$ является связньм множеством, содержащим точку $\bar{h}(\bar{x})$, и принадлежит прямой линии $w^{s}(\bar{h}(\bar{x}))$, то образ при отображении $\bar{h}$, по крайней мере, одной из компонент множества $l_{\bar{x}}^{s} \backslash \bar{x}$ является ограниченным подмножеством прямой линии $w^{s}(\bar{h}(\bar{x}))$. Это противоречит тому, что отображение $\bar{h}$ является собственным.

Шаг 3. Покажем, что отображение $h$ является гомеоморфизмом. Из вьше доказанного следует, что

1) любая точка $\bar{x}$ плоскости $\mathbb{R}^{2}$ является единственной точкой пересечения $l_{\bar{x}}^{s} \cap l_{\bar{x}}^{u}$ и $w^{s}(\bar{x}) \cap w^{u}(\bar{x})$;

2 ) ограничение отображения $\bar{h}$ на каждую из кривых $l_{\bar{x}}^{s}, l_{\bar{x}}^{u}$ является взаимнооднозначным отображением на $w^{s}(\bar{h}(\bar{x})), w^{u}(\bar{h}(\bar{x}))$ соответственно.

Из свойств 1) и 2) следует, что отображение $\bar{h}: \mathbb{R}^{2} \rightarrow \mathbb{R}^{2}$ является взаимнооднозначньм.

Тогда отображение $h: M_{i}^{2} \rightarrow M_{i}^{2}$ является взаимнооднозначным и непрерывньм отображением и, как легко видеть, является гомеоморфизмом.

Лемма полностью доказана.

Работа была в основном написана, когда первый и третий авторы находились в университетах городов Нанта и Ренна соответственно в марте-июне 2004 года. Они благодарят CNRS, которьй сделал эти пребывания возможньми. Они хотели бы также поблагодарить Франсуа Лауденбаха и Андрея Пажитнова, Вадима Каймановича и Антона Зорича за очень плодотворные обсуждения и гостеприимство.

\section{СПИСОК ЦИТИРОВАННОЙ ЛИТЕРАТУРЫ}

[1] Franks J. Anosov diffeomorphisms // Global Analysis. Proc. of Symposia in Pure Math. V. 14. Providence, RI: Amer. Math. Soc., 1970. P. 61-94.

[2] Newhouse S. On codimension one Anosov diffeomorphisms // Amer. J. Math. 1970. V. 92. № 3. P. $761-770$.

[3] Hiraide K. A simple proof of the Franks-Newhouse theorem on codimension one Anosov diffeomorphisms // Ergodic Theory Dynamical Systems. 2001. V. 21. P. 801-806.

[4] Grines V., Zhuzhoma E. On structurally stable diffeomorphisms with codimension one expanding attractors // Trans. Amer. Math. Soc. 2005. V. 357. № 2. P. 617-667.

[5] Гринес В. З., Ж Кужома Е. В. О грубых диффеоморфизмах с растягивающимися аттракторами и сжимающимися репеллерами коразмерности один // Докл. РАН. 2000. Т. 374. №6. C. $735-737$.

[6] Katok A., Hassenblatt B. Introduction to the Modern Theory of Dynamical Systems. Encyclopedia of Math. and its Applications. Cambridge: Cambridge Univ. Press, 1994.

[7] Robinson C. Dynamical Systems, Stability, Symbolic Dynamics, and Chaos. 2nd edition. Boca Raton, FL: CRC Press, 1999.

[8] Smale S. Differentiable dynamical systems // Bull. Amer. Math. Soc. 1967. V. 73. №1. P. $741-817$.

[9] Abraham R., Smale S. Nongenericity of $\Omega$-stability // Global Analysis. Proc. of Symposia in Pure Math. V. 14. Providence, RI: Amer. Math. Soc., 1970. P. 5-8.

[10] Williams R. F. One-dimensional non-wandering sets // Topology. 1967. V. 6. P. 473-487.

[11] Pesin Y. Dimension Theory in Dynamical Systems, Contemporary Views and Applications. Chicago: Univ. of Chicago Press 1997.

[12] Shub M., Sullivan D. Homology theory and dynamical systems // Topology. 1975. V. 14. P. $109-132$.

[13] Bothe H. The ambient structure of 1-dimensional hyperbolic structure in 3-manifolds. V. 81. Berlin: Inst. Math., Akad. Wissensch. DDR, 1981. 
[14] Jiang B., Ni Y., Wang S. 3-manifolds that admit knotted solenoids as attractors // Trans. Amer. Math. Soc. 2004. V. 356. № 11. P. 4371-4382.

[15] Hempel J. 3-Manifolds. Ann. of Math. Studies. V. 86. Princeton, NJ: Princeton Univ. Press, 1976.

[16] Плыкин Р. В. О топологии базисных множеств диффеоморфизмов С. Смейла // Матем. сб. 1971. T. 84. № 2. C. 301-312.

[17] Williams R. F. Expanding attractors // Publ. Math. IHES. 1974. V. 43. P. 169-203.

[18] Kaplan J., Mallet-Paret J. Yorke J. The Lyapunov dimension of nowhere differentiable attracting torus // Ergodic Theory Dynamical Systems. 1984. V. 4. № 2. P. 261-281.

[19] Pixton D. Wild unstable manifolds // Topology. 1977. V. 16. P. 167-172.

[20] Bonatti C., Grines V. Knots as topological invariants for gradient-like diffeomorphisms of the sphere $S^{3} / /$ J. Dynamical Control Systems. 2000. V. 6. № 4. P. 579-602.

[21] Аносов Д.В. Об одном классе инвариантных множеств гладких динамических систем // Тр. 5-й Междунар. конф. по нелинейным колебаниям. Качественные методы.. Киев 1970. Т. 2. C. $39-45$.

[22] Bowen R. Periodic points and measures for axiom A-diffeomorphisms // Trans. Amer. Math. Soc. 1971. V. 154. P. 377-397.

[23] Hurewicz W., Wallman H. Dimension Theory. Princeton, NJ: Princeton Univ. Press, 1984.

[24] Moise Ed. E. Geometric Topology in Dimensions 2 and 3. Graduate Texts in Math. V. 47. New York-Heidelberg: Springer-Verlag, 1977.

[25] Engelking R. General Topology. Berlin: Helderman-Verlag, 1989.

[26] Knezer H. Regulare Kurvenscharen auf Ringflachen // Math. Ann. 1924. V. 91. P. 135-154.

[27] Aranson S., Grines V., Kaimanovich V. Classification of supertransitive 2-webs on surfaces // J. Dynamical Control Systems. 2003. V. 9. № 4. P. 455-468.

[28] Franks J. Anosov diffeomorphisms on tori // Trans. Amer. Math. Soc. 1969. V. 145. P. $117-124$.

(В.З. Гринес) Нижегородская государственная сельскохозяйственная академия 